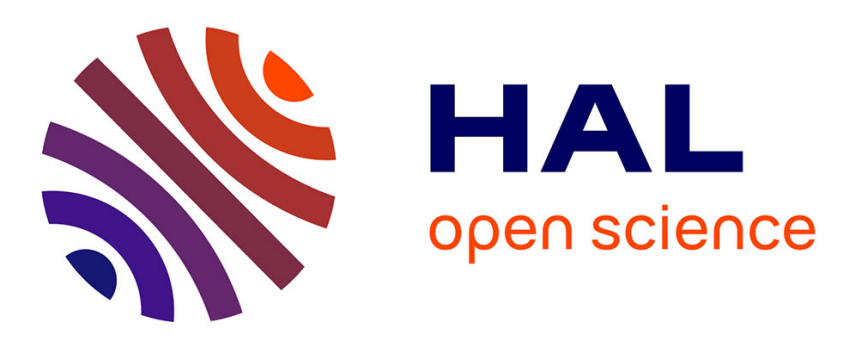

\title{
Dispersive Nature of High Mach Number Collisionless Plasma Shocks: Poynting Flux of Oblique Whistler Waves
}

David Sundkvist, V Krasnoselskikh, S.D. Bale, S.J. Schwartz, J Soucek, F Mozer

\section{To cite this version:}

David Sundkvist, V Krasnoselskikh, S.D. Bale, S.J. Schwartz, J Soucek, et al.. Dispersive Nature of High Mach Number Collisionless Plasma Shocks: Poynting Flux of Oblique Whistler Waves. Physical Review Letters, 2012, 108 (2), pp.025002 10.1103/PhysRevLett.108.025002 . insu-01259308

\section{HAL Id: insu-01259308 https://hal-insu.archives-ouvertes.fr/insu-01259308}

Submitted on 20 Jan 2016

HAL is a multi-disciplinary open access archive for the deposit and dissemination of scientific research documents, whether they are published or not. The documents may come from teaching and research institutions in France or abroad, or from public or private research centers.
L'archive ouverte pluridisciplinaire HAL, est destinée au dépôt et à la diffusion de documents scientifiques de niveau recherche, publiés ou non, émanant des établissements d'enseignement et de recherche français ou étrangers, des laboratoires publics ou privés. 


\title{
Dispersive Nature of High Mach Number Collisionless Plasma Shocks: Poynting Flux of Oblique Whistler Waves
}

\author{
David Sundkvist, ${ }^{1, *}$ V. Krasnoselskikh, ${ }^{2}$ S. D. Bale, ${ }^{1}$ S. J. Schwartz ${ }^{3}$ J. Soucek, ${ }^{4}$ and F. Mozer ${ }^{1}$ \\ ${ }^{1}$ Space Sciences Laboratory, University of California, Berkeley, California, USA \\ ${ }^{2} L P C 2 E$, CNRS, Orléans, France \\ ${ }^{3}$ Blackett Laboratory, Imperial College London, London SW7 2AZ, United Kingdom \\ ${ }^{4}$ Institute of Atmospheric Physics, Academy of Sciences of the Czech Republic, Prague, Czech Republic
} (Received 28 February 2011; published 11 January 2012)

\begin{abstract}
Whistler wave trains are observed in the foot region of high Mach number quasiperpendicular shocks. The waves are oblique with respect to the ambient magnetic field as well as the shock normal. The Poynting flux of the waves is directed upstream in the shock normal frame starting from the ramp of the shock. This suggests that the waves are an integral part of the shock structure with the dispersive shock as the source of the waves. These observations lead to the conclusion that the shock ramp structure of supercritical high Mach number shocks is formed as a balance of dispersion and nonlinearity.
\end{abstract}

DOI: 10.1103/PhysRevLett.108.025002

PACS numbers: 52.35.Tc, 94.05.-a, 95.30.Qd

Shock plasma waves are ubiquitous in our Universe. They play an important role in redistributing kinetic energy in supersonic flow into plasma thermal energy and energetic particles. In particular, Earth's bow shock defines the boundary between the supersonic solar wind plasma and the subsonic region of the near-Earth space environment.

Despite the absence of collisions, low Mach number collisionless shocks are treated as steady state fast magnetosonic nonlinear waves or discontinuities in a dissipative MHD approximation. This allows one to determine the asymptotic state of the plasma and magnetic field across a shock, by using the Rankine-Hugoniot conservation laws. Any deviation from MHD such as two-fluid or kinetic descriptions results in the appearance of dispersive effects.

When the Mach number of the shock increases past a critical Mach number, $M_{\text {crit }}$, inferred in the frame of a MHD description, neither resistive nor viscous effects can provide sufficient dissipation to sustain a stationary shock transition [1]. For these so-called supercritical shocks the major dissipation mechanism is related to reflected ions [2-4] that require a kinetic description.

It is well known that a subcritical shock has a nonlinear whistler wave train upstream of its front $[5,6]$. The major transition of such a dispersive shock, the ramp, behaves as the largest peak of the whistler precursor wave package [7-10]. The presence of whistler or fast magnetosonic precursor wave trains in supercritical shocks as well was experimentally established in [11-13]. These whistler waves have rather large amplitudes, and their role in energy transformation and redistribution between different particle populations and in the formation of the shock front structure is still an open question. Often the precursor waves are almost phase standing in the shock frame. However, their group velocity can still be greater than zero in the shock reference frame, which would allow energy flow in the form of Poynting flux to be emitted towards the upstream of the shock transition. In this Letter we address this problem and present the first direct measurement of the Poynting flux of the upstream whistler waves.

It has been suggested that the shock front structure of quasiperpendicular supercritical shocks is formed similarly to that of subcritical shocks [14]. The observed dynamic features of shocks have also been studied extensively using computer particle-in-cell (PIC) or hybrid simulations, often with focus on the precursor wave activity and reflected ions [15-17]. From a kinetic viewpoint, however, it may be argued that the shock-reflected ions change the physical picture and that the principal scales, temporal and spatial, could be determined by the characteristics of the reflected ion population [18]. Upstream waves can then be generated due to counterstreaming ions and electrons in the shock front region, forming unstable particle distributions with respect to some wave modes $[16,17,19]$. While this is probably the case for some higher frequency waves, our analysis below leads to the conclusion that the source of the upstream low frequency whistler waves is related to the presence of the nonlinear ramp transition, emitting smaller scale dispersive waves towards the upstream flow.

The existence of phase-standing upstream whistler waves depends on the value of the upstream flow speed Mach number relative to the phase velocity. If the Mach number of the shock does not exceed the whistler critical Mach number $M_{w}=V_{w, \max } / V_{A}=1 / 2 \sqrt{m_{i} / m_{e}} \cos \theta_{B n}$, the highest possible phase velocity, then phase-standing (linear) whistler wave trains can exist upstream of the shock $[9,14]$. In the above equation $V_{A}$ is the Alfvén speed and $\theta_{B n}$ is the angle between the upstream magnetic field and the normal to the shock.

Below we establish the energy source of the waves by calculating the Poynting flux of the waves in the normal incidence frame (NIF) of the shock, using multisatellite 
Cluster data from crossings of Earth's bow shock [20-22]. Two events with supercritical Alfvénic Mach numbers are analyzed. In both cases it is found that the shocks show dispersive behavior with upstream Poynting flux.

Poynting flux is not a Lorentz invariant and therefore depends on the frame of reference. To evaluate the value and direction of the Poynting flux with respect to the shock we transform the electric field to the NIF. The normal $\hat{\mathbf{n}}=$ $+\hat{\mathbf{x}}$, which also serves as the $x$-coordinate direction in the NIF system, is obtained by four-spacecraft timing, $\hat{\mathbf{z}}$ is the direction of maximum varying magnetic field obtained from a minimum variance analysis, and $\hat{\mathbf{y}}$ is the direction of the convection electric field which completes the righthanded system.

The transformation from the spacecraft frame to the NIF is given by $\mathbf{E}_{\mathrm{NIF}}=\mathbf{E}_{\mathrm{sc}}+\mathbf{v} \times \mathbf{B}$. The total velocity in the boost is $\mathbf{v}=\mathbf{v}_{\mathrm{sh}}+\mathbf{v}_{\mathrm{NIF}}$, where $\mathbf{v}_{\mathrm{sh}}=\boldsymbol{v}_{\mathrm{sh}} \hat{\mathbf{n}}$ is the shock velocity, $\mathbf{v}_{\mathrm{NIF}}=\hat{\mathbf{n}} \times\left(\mathbf{v}_{u} \times \hat{\mathbf{n}}\right)$ is the NIF velocity, and $\mathbf{v}_{u}$ is the solar wind velocity.

A general shift of reference frame, coordinate transformation, and evaluation of the complete Poynting vector requires knowledge of the full six-dimensional electromagnetic field (three electric and three magnetic components). The Cluster spacecraft, however, only measures the two components of the electric field in the spin plane of the spacecraft, while the third component normal to the spin plane is not measured. To reconstruct the third component we use the assumption that for the wave electric and magnetic fields the condition $\mathbf{E} \cdot \mathbf{B}=0$ holds. While this is most probably not a true condition for the cross shock (dc) electric field, it holds well for whistler wave electric fields at lower frequencies.

We study two quasiperpendicular high Mach number shocks encountered by the Cluster multi-spacecraft mission [20]. The first shock was observed around 04:53:40 universal time (UT) on 20 January 2003, and the second around 07:07:00 UT on 24 January 2001. We use data primarily from the EFW (electric field), FGM (dc magnetic field), and STAFF (wave magnetic field) instruments [20] from spacecraft 2 (for the 2003 shock) and spacecraft 3 (for the 2001 shock). The shock normal $\hat{\mathbf{n}}$ is established by assuming a planar shock and using the time of crossing of the four spacecraft and their relative positions [23].

The first shock analyzed had an upstream $\theta_{B n} \sim 85^{\circ}$ and an Alvénic Mach number $M_{A} \sim 5.5$. The electric and magnetic fields in the shock front region are characterized by waves, with stronger amplitudes closer to the ramp; see Fig. 1. The waves have frequencies $f_{\mathrm{cp}}<f$, where $f_{\mathrm{cp}} \sim$ $0.1 \mathrm{~Hz}$ is the proton gyrofrequency, and right-handed polarization looking along the magnetic field vector, and thus belong to the magnetosonic or whistler mode. The direction of the wave vector $\hat{\mathbf{k}}$ was determined by the Means method [24], which uses the imaginary part of the threedimensional magnetic field spectral matrix. The angle $\theta_{k B}$ between the wave vector and the local ambient magnetic

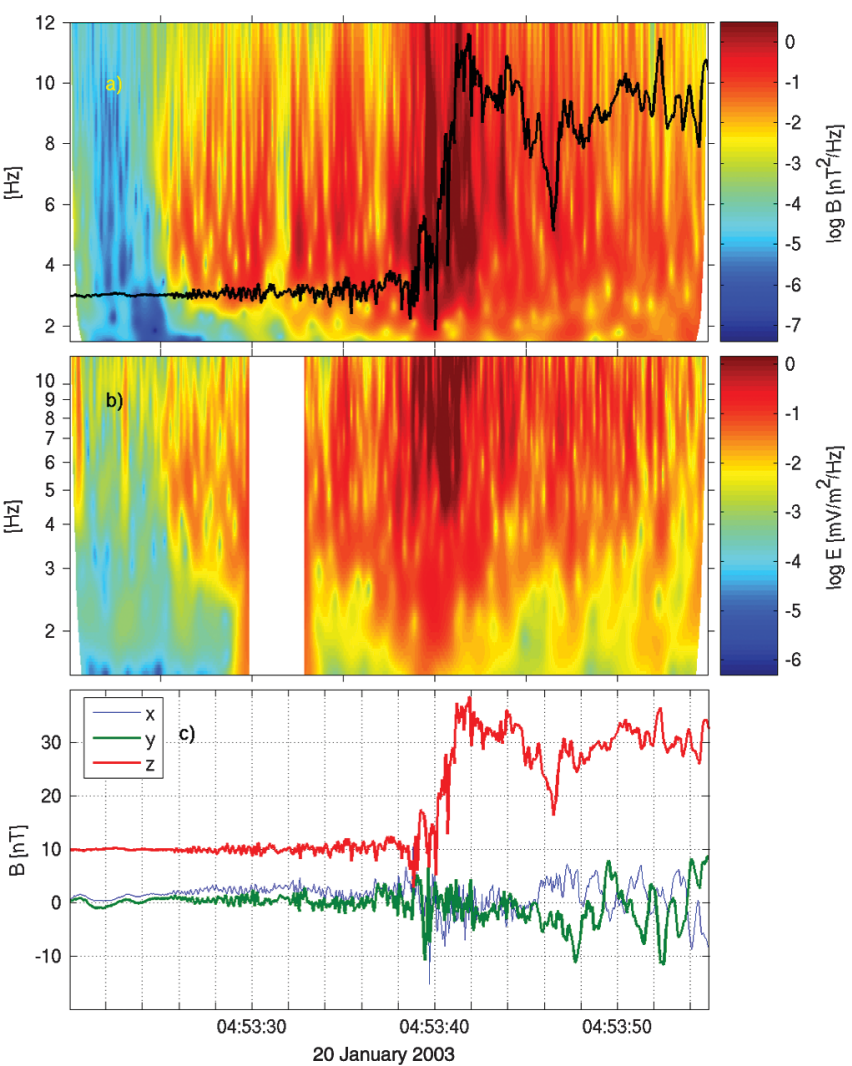

FIG. 1 (color). Magnetic and electric fields in the normal incidence frame (NIF) of a high Mach number shock. (a) Power spectra of the magnetic field (STAFF). The black line is the dc total magnetic field, included to show the waves in relation to the shock ramp structure. (b) Power spectra of the electric field (EFW). The data gap is due to instrumental interference. (c) The magnetic field in NIF coordinates $B_{\mathrm{NIF}}$.

field is shown as a function of frequency in Fig. 2(b). The average value $\left\langle\theta_{k B}\right\rangle$ in the shock front region is $\sim 10^{\circ}-50^{\circ}$ (right-hand scale). The whistler waves are thus oblique with respect to the local magnetic field, as well as to the shock normal. The angle increases continuously as the shock front is approached and $\theta_{k B} \rightarrow 90^{\circ}$ at the ramp, reflecting the quasiperpendicular nature of the shock. This smooth transition stresses the nature of the shock as a dispersive nonlinear whistler wave.

Since Poynting flux is a second-order quantity the electric and magnetic fields in the NIF were wavelet transformed (Morlet width 5.36) and the cross product $\mathbf{S}_{f}=1 / \mu_{0} \mathbf{E}_{f} \times \mathbf{B}_{f}$ formed in frequency space. The calculated Poynting flux is therefore distributed in both time and frequency. The projection of the Poynting flux distribution along the magnetic field $S_{\|}=\mathbf{S}_{f} \cdot \mathbf{B}_{0} /\left|\mathbf{B}_{0}\right|$ using an instantaneous value of $\mathbf{B}_{0}$ is plotted in Fig. 2(a), where the colors red (upstream) and blue (downstream) show the direction of the flux. We note that in the front region of the shock the Poynting flux is everywhere directed upstream (red), away from the shock. In the downstream area there is a mixture of blue, green, and red, where there is more 


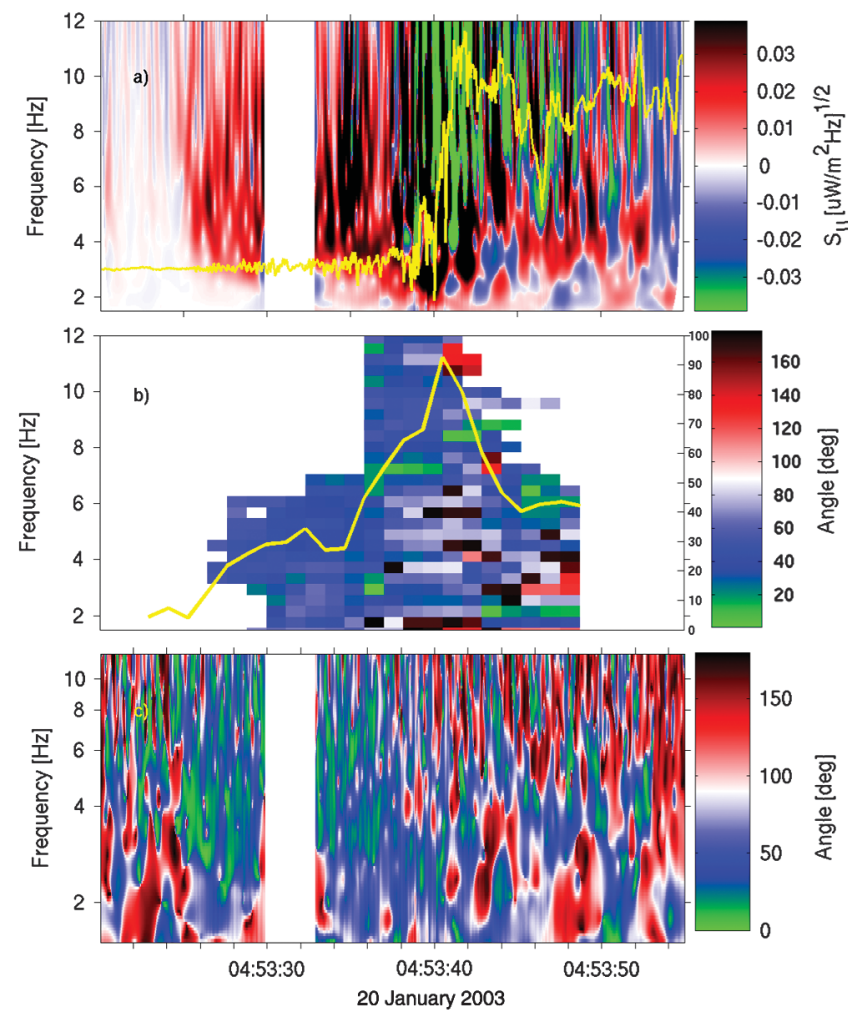

FIG. 2 (color). Poynting flux in the NIF of the same shock as in Fig. 1. (a) Poynting flux $S_{\|}$projected on the local $\mathbf{B}_{0}$ in the NIF, where red corresponds to the upstream flux away from the shock. (b) Angle $\theta_{k, B}$ between $\hat{\mathbf{k}}$ and magnetic field $\mathbf{B}_{0}$. The yellow line represents the average over all frequencies (right-hand scale). (c) Angle between Poynting flux $\mathbf{S}$ and $\mathbf{B}_{0}$.

turbulence and the waves are no longer coherent. The upstream and slightly oblique direction of the Poynting flux is further quantified in the instantaneous angle $\theta_{S, B}$ between the Poynting flux and the ambient magnetic field, plotted in Fig. 2(c). Figure 3 shows the Poynting flux along the spacecraft trajectory, with integrated power over frequencies corresponding to the waves in Fig. 2(a), $2<f<$ $10 \mathrm{~Hz}$. In this figure the slope is the important characteristic. Positive slope means Poynting flux carried upstream, and negative slope downstream. From the figure it is evident that the source of the Poynting flux is associated with the shock ramp. The data gap and associated plateau are due to instrumental interference.

Another important characteristic established is that the Poynting flux direction is oblique with respect to the shock normal as well as the background magnetic field. This can be explained by analyzing how the phase velocity for whistler waves depends on this angle. The phase velocity of a wave propagating in the plane of the shock normal $\hat{\mathbf{n}}$ and background magnetic field $\mathbf{B}_{0}$, having an angle $\alpha$ with respect to the shock normal, is $V_{\mathrm{ph}}=\frac{1}{2} \times$ $\sqrt{m_{i} / m_{e}} \cos \left(\theta_{B n}-\alpha\right)$. Its projection on the direction of the shock normal is $V_{\mathrm{ph}, \hat{\mathbf{n}}}=$ $V_{\mathrm{ph}} \cos \alpha=\frac{1}{2} \sqrt{m_{i} / m_{e}} \cos \left(\theta_{B n}-\alpha\right) \cos \alpha$. Its maximum

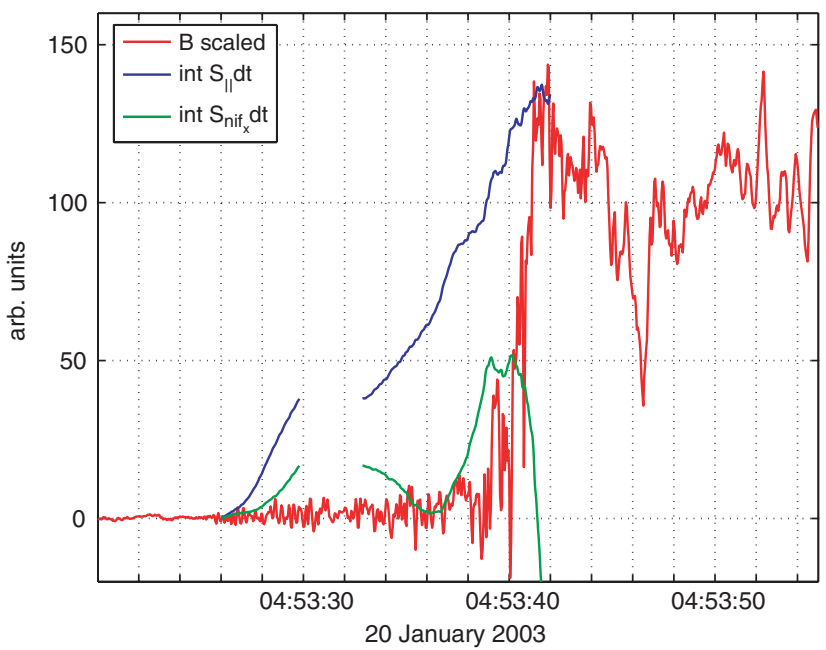

FIG. 3 (color). Poynting flux integrated along the spacecraft trajectory. The blue line is a projection along the ambient magnetic field $\int S_{\|} d t$ and the green line is a projection on the shock normal $\int \mathbf{S} \cdot \hat{\mathbf{n}} d t$. The red line shows the scaled magnetic field $B_{0}$ for reference.

value can be found to be equal to $\max \left(V_{p h, \hat{\mathbf{n}}}\right)=$ $\frac{1}{4} \sqrt{m_{i} / m_{e}}\left(1+\cos \theta_{B n}\right)$, thus the projected phase speed can be larger than the whistler critical Mach number given above. The above analysis also explains the observation of oblique whistler wave trains found in computer simulations of purely perpendicular shocks [16]. So even in the case of shocks having Mach numbers larger than the whistler critical Mach number, whistler waves oblique with respect to the shock normal can remain quasistanding.

The second analyzed shock crossing on 24 January 2001 is shown in Fig. 4. This is a reforming high Mach number shock $\left(M_{A} \sim 11\right)$ and has been analyzed in detail in [25]. Both shocks shown in this Letter were analyzed using wavelet as well as fast Fourier transform (FFT) dynamic spectra techniques. We present the second shock using the FFT analysis, to show that the conclusions are not technique dependent. The upstream whistler waves, Figs. 4(a) and 4(b), again have an overall Poynting flux upstream, away from the shock in the normal incidence frame, evident from the red and yellow (upstream) colors of $S_{\|}$ [Figs. 4(c)-4(f)]. For this shock the ambient magnetic field was directed in the opposite direction, so that $180^{\circ}$ (red) means upstream in Fig. 4.

In this Letter we analyzed oblique whistler waves in the region upstream of the front of collisionless quasiperpendicular high Mach number shocks, using data from the Cluster mission. The power flux given by the Poynting vector shows that they carry energy over a broad frequency range from the shock ramp towards the upstream solar wind, starting from the position of the shock front. This is consistent with a theoretical model $[9,10,14]$ that considers the shock steepening to be balanced by the effect of dispersion in addition to dissipation. As the shock steepens, 

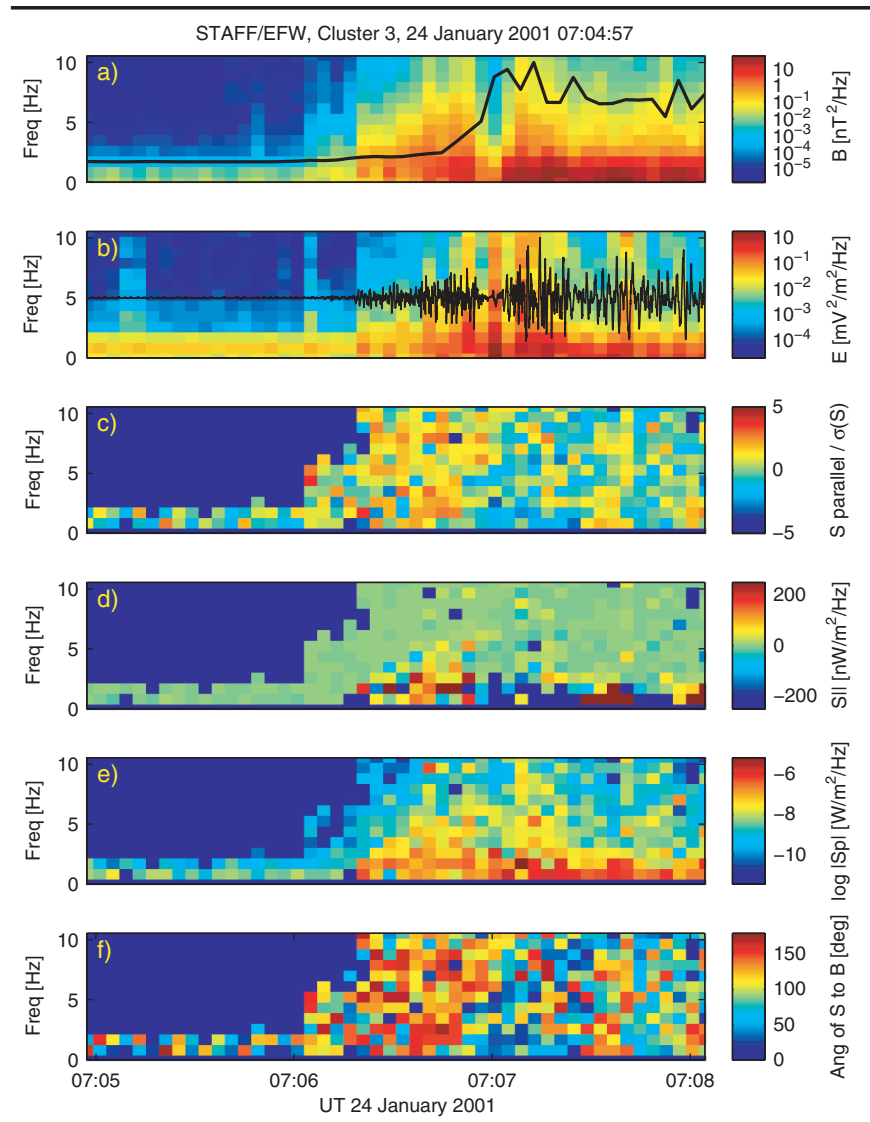

FIG. 4 (color). Poynting flux derived from electric and magnetic fields for a high Mach number shock. (a) Wave magnetic field and averaged $B_{0}$. (b) Wave electric field. (c) $S_{\|}$normalized by its standard deviation (yellow and red correspond to upstream flux). (d) $S_{\|}$. (e) $\log _{10} S_{\|}$. (f) Angle of $S$ to $\mathbf{B}_{0}$ (red meaning upstream).

nonlinearities transfer energy to shorter wavelengths of the spectrum, and is ultimately carried away from the shock as dispersive whistler wave trains. This is consistent with our analysis of the upstream Poynting flux of the precursor waves. A future study will present statistics on what fraction of the energy is carried away by Poynting flux. This analysis demonstrates that for high Mach number shocks, dispersive effects are important for the formation and stability of the shock front. Since the whistler waves are strongly damped upstream of the shock, we infer that they can play the role of an intermediate step in the energy repartition problem, with the energy ultimately being dissipated through wave-particle interaction.

*sundkvist@ssl.berkeley.edu

[1] F. V. Coroniti, J. Plasma Phys. 4, 265 (1970).
[2] M. M. Leroy, D. Winske, C.C. Goodrich, C.S. Wu, and K. Papadopoulos, J. Geophys. Res. 87, 5081 (1982).

[3] G. Paschmann, N. Sckopke, S. J. Bame, and J. T. Gosling, Geophys. Res. Lett. 9, 881 (1982).

[4] N. Sckopke, G. Paschmann, S. J. Bame, J. T. Gosling, and C. T. Russell, J. Geophys. Res. 88, 6121 (1983).

[5] R. Z. Sagdeev, Rev. Plasma Phys. 4, 23 (1966).

[6] M. M. Mellott, Geophysical Monograph Series 35, 131 (1985).

[7] V. I. Karpman, Sov. Phys. Tech. Phys. 8, 715 (1964).

[8] C.F. Kennel, J.P. Edmiston, and T. Hada, Geophysical Monograph Series 34, 1 (1985).

[9] A. A. Galeev, V. V. Krasnoselskikh, and V. V. Lobzin, Sov. J. Plasma Phys. 14, 1192 (1988).

[10] V. V. Krasnoselskikh, B. Lembège, P. Savoini, and V. V. Lobzin, Phys. Plasmas 9, 1192 (2002).

[11] M. A. Balikhin, T. D. de Wit, H. S. C. K. Alleyne, L. J. C. Woolliscroft, S. N. Walker, V. Krasnoselskikh, W. A.C. Mier-Jedrzejeowicz, and W. Baumjohann, Geophys. Res. Lett. 24, 787 (1997).

[12] V. V. Krasnoselskikh, T. Vinogradova, M. A. Balikhin, H. S. C. Alleyne, A. K. Pardaens, L. J. C. Woolliscroft, S. I. Klimov, A. Petrukovich, W. A.C. MierJedrzejowicz, and D. J. Southwood, Adv. Space Res. 11, 15 (1991).

[13] M. Oka et al., Geophys. Res. Lett. 33, 24104 (2006).

[14] A. A. Galeev, C. F. Kennel, V. V. Krasnoselskikh, and V. V. Lobzin, in Proceedings of the Joint Varenna-Abastumani International School Workshop on Plasma Astrophysics, Varenna, Italy, 1988, edited by T. D. Guyenne (European Space Agency, Noordwijk, The Netherlands, 1988), Vol. ESA SP 285, pp. 165-171.

[15] P. Hellinger and A. Mangeney, J. Geophys. Res. 102, 9809 (1997).

[16] P. Hellinger, P. Trávníček, B. Lembège, and P. Savoini, Geophys. Res. Lett. 34, 14109 (2007).

[17] S. Matsukiyo and M. Scholer, J. Geophys. Res. (Space Phys.) 111, 6104 (2006).

[18] S. D. Bale, F. S. Mozer, and T. S. Horbury, Phys. Rev. Lett. 91, 265004 (2003).

[19] K. Papadopoulos, Geophysical Monograph Series 34, 59 (1985).

[20] C. P. Escoubet, R. Schmidt, and M. L. Goldstein, Space Sci. Rev. 79, 11 (1997).

[21] J. R. Wygant, M. Bensadoun, and F. S. Mozer, J. Geophys. Res. 92, 11109 (1987).

[22] S. D. Bale et al., Space Sci. Rev. 118, 161 (2005).

[23] G. Paschmann and P. W. Daly, Analysis Methods for MultiSpacecraft Data (European Space Agency, Noordwijk, The Netherlands, 1998).

[24] J. D. Means, J. Geophys. Res. 77, 5551 (1972).

[25] V. V. Lobzin, V. V. Krasnoselskikh, J. Bosqued, J. Pinçon, S. J. Schwartz, and M. Dunlop, Geophys. Res. Lett. 34, 5107 (2007). 